\title{
Resultados del Tratamiento del Hepatoblastoma en Los Andes Ecuatorianos
}

\author{
Results of the Treatment of Hepatoblastoma in the Ecuadorian Andes
}

\author{
Enmanuel Guerrero ${ }^{1,2,3}$; Raúl Alvarado; Pablo Monsalve' $^{1}$; Eulalia Peñafiel ${ }^{1}$; \\ Tatiana Díaz2; Galo Duque ${ }^{3,4}$; Carlos Manterola ${ }^{3,5}$ \& Lorena Albarracín $^{3}$
}

GUERRERO, E.; ALVARADO, R.; MONSALVE, P.; PEÑAFIEL, E.; DÍAZ, T.; DUQUE, G.; MANTEROLA, C.; ALBARRACÍN, L. Resultados del tratamiento del hepatoblastoma en Los Andes ecuatorianos. Int. J. Morphol., 39(6):1737-1742, 2021.

RESUMEN: El hepatoblastoma (HB), es una neoplasia maligna, que se origina en el hígado. La supervivencia (SV) depende de la extensión de avance de la enfermedad. El objetivo de este estudio fue determinar diferencias en la SV actuarial global (SVAG) y libre de enfermedad (SVLE) en pacientes con HB, según la extensión de su enfermedad. Serie de casos con seguimiento. Se incluyeron pacientes de entre 4 y 160 meses de edad tratados en un centro oncológico de Los Andes ecuatorianos (2000-2019). Las variables resultado fueron: lóbulo afectado, metástasis pulmonar, infiltración vascular, estadio PRETEXT, riesgo, histología, niveles de alfafetoproteína (AFP), remisión completa (RC), SVAG y SVLE. Se utilizó estadística descriptiva y analítica (Chi2, exacto de Fisher y corrección por continuidad). Se realizaron análisis de SV con curvas de Kaplan Meier y log-rank. Fueron estudiados 28 pacientes (53,6 $\%$ hombres), con una mediana de edad de 40 meses. Se verificaron metástasis pulmonares e infiltración vascular en el 25,0 \% y 35,7 \% de los casos respectivamente. La histología, estadio clínico y riesgo alto fueron mayoritariamente tipo epitelial (42,8 \%), PRETEXT II $(50,0 \%)$ y riesgo alto $(67,8 \%)$ respectivamente. La media de AFP al diagnóstico fue $1055712 \mathrm{ng} / \mathrm{ml}$ y 9 pacientes alcanzaron RC. La SVAG y SVLE general a 19 años fue 33,1 \% y 26,0 \% respectivamente.Según su extensión, la SVAG y la SVLE para los pacientes de riesgo estándar y alto fueron $50,0 \%$ y $25,4 \%(\mathrm{p}=0,148)$; y 50,0 \% y 14,7\% ( $\mathrm{p}=0,037)$ respectivamente. La SVAG y SVLE verificadas son menores a las reportadas en otros estudios. La SVLE según su extensión, presentó diferencia significativa, sin embargo, este resultado debe ser tomado con cautela debido al número pequeño de pacientes.

PALABRAS CLAVE: Hepatoblastoma; Supervivencia; Quimioterapia; Cirugía.

\section{INTRODUCCIÓN}

El hepatoblastoma (HB) es el tumor maligno del hígado más frecuente de la infancia (Kiruthiga et al., 2018); se presenta sobre todo entre el primer y segundo año (Sharma $e t$ al., 2017). Tiene una incidencia de 0,19 pacientes por cada 100.000 niños en Estados Unidos (Feng et al., 2019); y según el registro de tumores de nuestra institución, ocupa el séptimo lugar entre los tumores pediátricos, con una incidencia de 0,4 casos por cada 100.000 niños (Martinez et al., 2015).

La etiología del HB es desconocida, aunque podría asociarse con bajo peso al nacer, tabaquismo materno y algunos síndromes, como el de Beckwith-Wiedemann (Feng et al.).

El diagnóstico se plantea ante la presencia de masa tumoral hepática asociada a elevación de alfafetoproteína (AFP)
(Davenport et al., 2012; Czauderna \& Garnier, 2018). No obstante lo cual, se puede recurrir a la toma de biopsias (Weldon et al., 2020); a la determinación de factor inhibidor de la migración de macrófagos, y de interleucina 25 , los que además aportan al pronóstico de la enfermedad (Guo et al., 2017).

Antes de planificar tratamiento, se recomienda estadificar a los pacientes utilizando PRETEXT (Sociedad Internacional de Oncología Pediátrica para Tumores Hepáticos (SIOPEL)); para definir extensión de enfermedad en riesgo estándar (PRETEXT I, II, III sin metástasis) y riesgo alto (PRETEXT IV con o sin metástasis) (Kremer et al., 2014). Posteriormente, suele indicarse quimioterapia neoadyuvante (QTNA) seguida de resección quirúrgica (Meyers et al., 2014; van Dalen et al., 2014; Sharma et al.; Liu et al., 2019).

\footnotetext{
${ }^{1}$ Instituto del Cáncer SOLCA, Cuenca, Cuenca, Ecuador.

${ }^{2}$ Facultad de Medicina, Universidad de Cuenca, Cuenca, Ecuador.

${ }^{3}$ Programa de Doctorado en Ciencias Médicas, Universidad de La Frontera, Temuco, Chile.

${ }^{4}$ Universidad del Azuay, Cuenca, Cuenca, Ecuador.

${ }^{5}$ Centro de Estudios Morfológicos y Quirúrgicos (CEMyQ), Universidad de La Frontera, Temuco, Chile.
} 
La supervivencia (SV) global es de alrededor del $75 \%$, y cuando existe enfermedad metastásica cercana al $20 \%$ (Feng et al.).

El objetivo de este estudio fue determinar diferencias en la SV actuarial global (SVAG) y libre de enfermedad (SVLE) en pacientes con HB, según extensión de enfermedad.

\section{MATERIAL Y MÉTODO}

Este artículo fue escrito siguiendo la iniciativa MInCir para el reporte de estudios observacionales descriptivos (Manterola \& Astudillo, 2013).

Diseño: Serie de casos con seguimiento.

Centro: El estudio fue realizado en un instituto oncológico de referencia.

Participantes: Fueron incluidos todos los pacientes con diagnóstico clínico o patológico de HB de entre 4 y 160 meses de edad tratados con QTNA y cirugía de forma. consecutiva; en el período enero de 2000 y mayo de 2019.Se excluyeron pacientes con información incompleta, y operados en otros hospitales.

Tamaño de muestra: Se trabajó con la totalidad del universo de pacientes con $\mathrm{HB}$, clasificados de acuerdo con la extensión de su enfermedad y sometidos a los tratamientos antes mencionados. Por ende, no se realizó cálculo del tamaño de la muestra.

Variables: Las variables resultado fueron SVAG y SVLE (general y según extensión de enfermedad), medidas en meses posteriores a la aplicación de las distintas terapias. Otras variables de interés fueron: biodemográficas, niveles de deshidrogenasa láctica (DHL) y AFP; lóbulo afectado, metástasis pulmonar, infiltración vascular, estadio PRETEXT, histología, complicaciones y recurrencia. En aquellos pacientes en los que la extensión de enfermedad no estaba claramente definida, se utilizaron las directrices actuales recomendadas por la SIOPEL (Meyers et al.; Liu et al.).

Seguimiento: El seguimiento mínimo fue de 12 meses.Este se realizó mediante el estudio de las historias clínicas, la base de datos institucional y los certificados de defunción obtenidos de la página Web del registro civil.

Estadísticas: El análisis exploratorio de los datos se reali- zó utilizando el paquete estadístico SSPS 23,0. Se aplicó estadística descriptiva con cálculo de proporciones, medidas de tendencia central y de dispersión. Se utilizó estadística analítica para comparación de promedios, con ttest para comparación de variables continuas; y Chi2 de Pearson o exacto de Fisher para comparación de variables categóricas. La SV se calculó y dibujó utilizando el método de Kaplan Meier y la prueba de log-rank para comparación de $\mathrm{SV}$ según extensión.

Principios éticos: Los procedimientos utilizados en los pacientes fueron realizados tras obtención de un consentimiento informado de los padres o tutores. La identidad de los pacientes se mantuvo oculta y sus datos fueron codificados. El estudio fue revisado y aprobado por el Comité de Investigación y Bioética de la institución donde se efectuó el estudio.

\section{RESULTADOS}

En el período estudiado, fueron tratados 28 pacientes con diagnóstico de HB. La mediana de edad al momento del diagnóstico fue 40 meses (4 a160 meses); y el $53,6 \%$ eran de sexo masculino.

Las localización más frecuentes del tumor fue bilateral y el lóbulo derecho (42,9 \% cada cual).En un 25,0 \% se verificó la existencia de metástasis pulmonares. En 10 casos $(35,7 \%)$, se constató infiltración de venas porta, cava o hepáticas. Un 25,0 \% de los casos se encontraban en estadio PRETEXT IV; y un 67,9 \% de los pacientes era de riesgo alto. En la mayoría de los casos el patrón histológico fue epitelial (42,9\%). Las características clínicas y patológicas se detallan en la Tabla I.

En la Tabla II, se describen las variaciones de los valores de la DHL y AFP; aumentados al diagnóstico y dentro de valores normales en los pacientes en RC.

En la Tabla III, se detalla el número de ciclos de quimioterapia pre y post quirúrgico, que variaron según repuesta clínica de cada paciente, pero dentro del intervalo recomendado por SIOPEL. También se puede observar el tiempo de recuperación postquirúrgico y el volumen tumoral de los diez pacientes que fueron intervenidos quirúrgicamente.

Luego de la administración de quimioterapia; nueve pacientes alcanzaron RC (Tabla IV); de éstos, un paciente tuvo recaída pulmonar a los 5 meses, siendo tratado con lobectomía pulmonar y 3 ciclos adicionales de qui- 
mioterapia. Seis de los pacientes que abandonaron el tratamiento $(86,0 \%)$, tenían enfermedad inicial avanzada (infiltración hepática bilateral, metástasis pulmonar e infiltración vascular). La mediana de SV de los tres pacientes que fallecieron fue 33 meses.

Los efectos adversos secundarios a quimioterapia y las complicaciones pos-quirúrgicas se observan en la Tabla V. Destacando las complicaciones respiratorias entre las causas de morbilidad postoperatoria (8/10 casos); y la anemia entre la causas de morbilidad posterior a quimioterapia $(94,7 \%)$.

La SVAG y SVLE general de esta serie fue 33,1\% y $26,0 \%$ respectivamente (Figs. 1 y 2). De acuerdo con la extensión de la enfermedad, la SVAG del grupo de pacientes de riesgo estándar y alto fue 50,0 \% y $25,4 \%$ respectivamente ( $\mathrm{p}=0,148)$ (Fig. 3). La SVLE en pacientes de riesgo estándar y alto fue $50,0 \%$ y $14,7 \%$ respectivamente ( $\mathrm{p}=0,037)$ (Fig. 4).

Tabla II. Valores de AFP y DHL de los pacientes en estudio.

\begin{tabular}{lcccc}
\hline \multicolumn{1}{c}{ Variables } & $\mathrm{N}^{\circ}$ casos & Media $\pm \mathrm{DE}$ & Mínimo & Máximo \\
\hline AFP (ng/ml) & & & & \\
Al diagnóstico & 28 & $1055712 \pm 1988431$ & 1,2 & 773200 \\
Postquirúrgico & 10 & $1032 \pm 2822$ & 3,1 & 8997 \\
Fin del & 10 & $18 \pm 38$ & 1,2 & 125 \\
DHL (U/L) & 27 & $945 \pm 974$ & 210 & 4607 \\
\hline
\end{tabular}

DE: Desviación estándar, AFP: Alfafetoproteína, DHL: Deshidrogenasa láctica.

Tabla III. Quimioterapia pre y postquirúrgico de los pacientes en estudio.

\begin{tabular}{lcccc}
\hline \multicolumn{1}{c}{ Variables } & $\mathrm{N}^{\circ}$ casos & Media $\pm \mathrm{DE}$ & Mínimo & Máximo \\
\hline QTNA* (ciclos) & 19 & $6,3 \pm 2,9$ & 1,0 & 10 \\
QTA* (ciclos) & 10 & $3,0 \pm 0,8$ & 2,0 & 5 \\
Recuperación PQ (días) & 10 & $10,1 \pm 3,6$ & 3,0 & 14 \\
Volumen tumoral $\left(\mathrm{cm}^{3}\right)$ & 10 & $182 \pm 234$ & 26,3 & 650 \\
\hline
\end{tabular}

DE: Desviación estándar, QTNA: Quimioterapia neoadyuvante, QTA: Quimioterapia adyuvante, PQ: Postquirúrgico, Quimioterapia administrada: *cisplatino $80 \mathrm{mg} / \mathrm{m}^{2}$ alternando con doxorrubicina $60 \mathrm{mg} / \mathrm{m}^{2}$ por 2 días y carboplatino $500 \mathrm{mg} / \mathrm{m}^{2}$ cada 15 días.

Tabla IV.Riesgo y condición luego del tratamiento administrado a los pacientes en estudio. $(\mathrm{N}=28)$

\begin{tabular}{lcccccc}
\hline \multirow{2}{*}{ Riesgo } & \multicolumn{5}{c}{ Condición post- tratamiento } \\
& RC & RP & Progresión & Abandono & Muerte & Total \\
\hline Estándar & 4 & 2 & 1 & 1 & 1 & 9 \\
Alto & 5 & 5 & 1 & 6 & 2 & 19 \\
Total & 9 & 7 & 2 & 7 & 3 & 28 \\
\hline
\end{tabular}

RC: Remisión completa. RP: Remisión parcial.
Tabla I. Características clínicas y patológicas de los pacientes en estudio $(\mathrm{N}=28)$.

\begin{tabular}{|c|c|c|}
\hline Variables & $\mathrm{N}^{\mathrm{o}} \operatorname{casos}$ & $\%$ \\
\hline \multicolumn{3}{|l|}{ Lóbulo afectado } \\
\hline Derecho & 12 & 42,9 \\
\hline Izquierdo & 4 & 14,2 \\
\hline Ambos & 12 & 42,9 \\
\hline \multicolumn{3}{|l|}{ Metástasis pulmonar } \\
\hline Sí & 7 & 25,0 \\
\hline No & 21 & 75,0 \\
\hline \multicolumn{3}{|l|}{ Infiltración vascular } \\
\hline Vena porta & 2 & 7,1 \\
\hline Venas cava y hepáticas & 6 & 21,4 \\
\hline Venas porta, cava y hepáticas & 2 & 7,1 \\
\hline Sin infiltración & 18 & 64,3 \\
\hline \multicolumn{3}{|l|}{ Estadio PRETEXT } \\
\hline I & 2 & 7,1 \\
\hline II & 14 & 50,0 \\
\hline III & 5 & 17,9 \\
\hline IV & 7 & 25,0 \\
\hline \multicolumn{3}{|l|}{ Extensión } \\
\hline Riesgo alto & 19 & 67,86 \\
\hline Ries go estándar & 9 & 32,14 \\
\hline \multicolumn{3}{|l|}{ Histología post quirúrgico y } \\
\hline Epitelial & 12 & 42,9 \\
\hline Mix to (epitelial y mesenquimal) & 3 & 10,7 \\
\hline Necrótico & 1 & 3,5 \\
\hline No realizado & 12 & 42,9 \\
\hline \multicolumn{3}{|l|}{ Procedimiento quirúrgico } \\
\hline Hepatectomía derecha & 9 & 32,1 \\
\hline Hepatect omía izquierda & 1 & 3,6 \\
\hline Toma de muestras para biopsia & 7 & 25,0 \\
\hline No se realizaron procedimientos & 11 & 39,3 \\
\hline
\end{tabular}

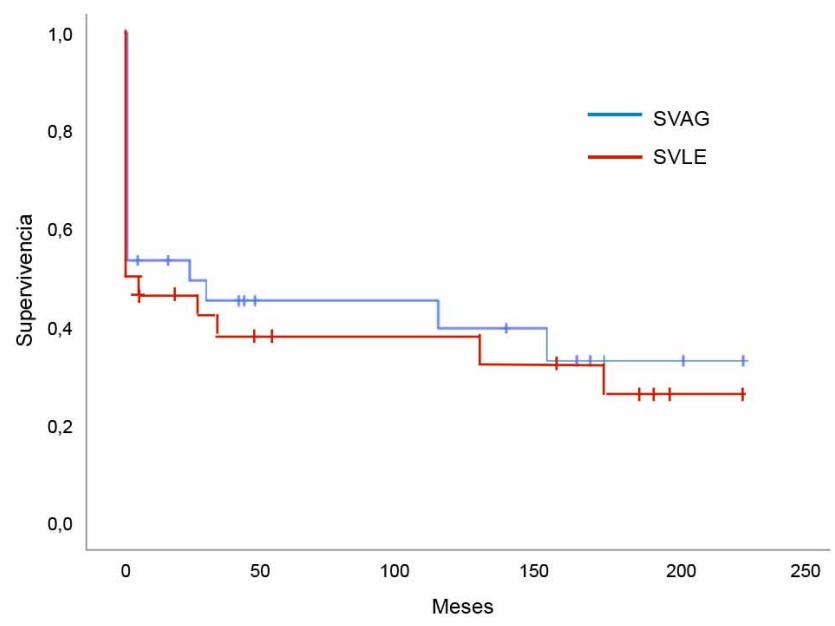

Fig. 1. SVAG para los pacientes con HB en estudio. 
Tabla V. Complicaciones postquirúrgicas y secundarias a la quimioterapia.

\begin{tabular}{clcc}
\hline Complicaciones & Variable & Frecuencia & $\%$ \\
\hline \multirow{4}{*}{$\begin{array}{c}\text { Postquirúrgico* } \\
(\mathrm{n}=10)\end{array}$} & Neumonía & 5 & 50,0 \\
& Shock hipovolémico & 5 & 50,0 \\
& Derrame pleural & 3 & 30,0 \\
& Digestivas & 3 & 30,0 \\
& Otras & 5 & 50,0 \\
& Anemia & 18 & 94,7 \\
& Náusea y vómitos & 17 & 89,4 \\
Post quimioterapia & Neutropenia & 16 & 84,2 \\
(n=19) & Neumonía & 10 & 52,6 \\
& Hepatopatía & 6 & 31,5 \\
& Bacteriemia/sepsis & 4 & 21,0 \\
& Alteración hidro- & 3 & 15,7 \\
& electrolítica & & \\
& Otras & 10 & 52,6 \\
\hline *No hubo mortalidad & secaria a la cirugía. & &
\end{tabular}

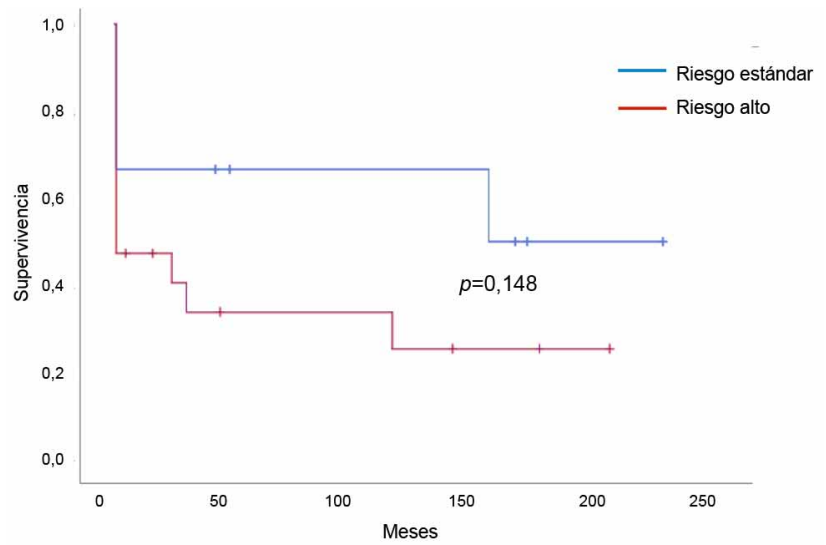

Fig. 2. SVLE para los pacientes con HB en estudio.

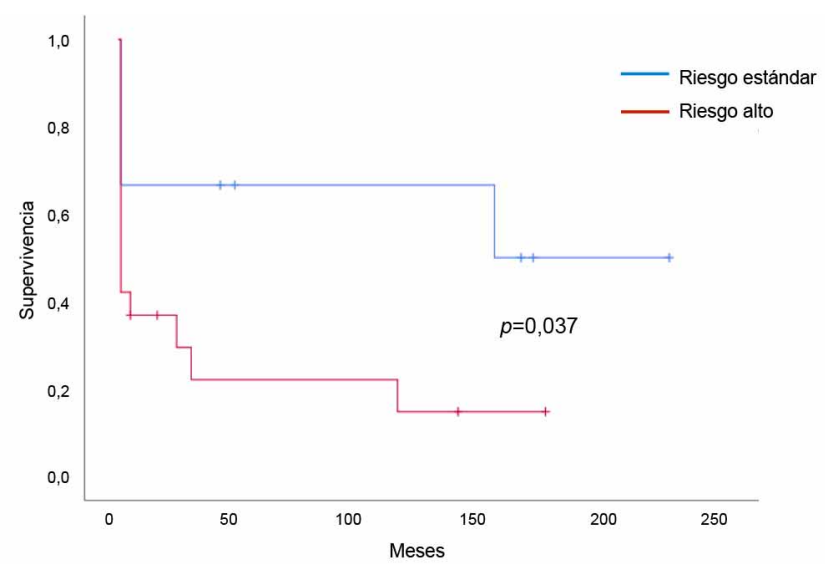

Fig. 3. SVAG para los pacientes de riesgo estándar y alto.

\section{DISCUSIÓN}

Novedad de la propuesta: Es un estudio novedoso por cuanto aporta información actualizada sobre las caracte-

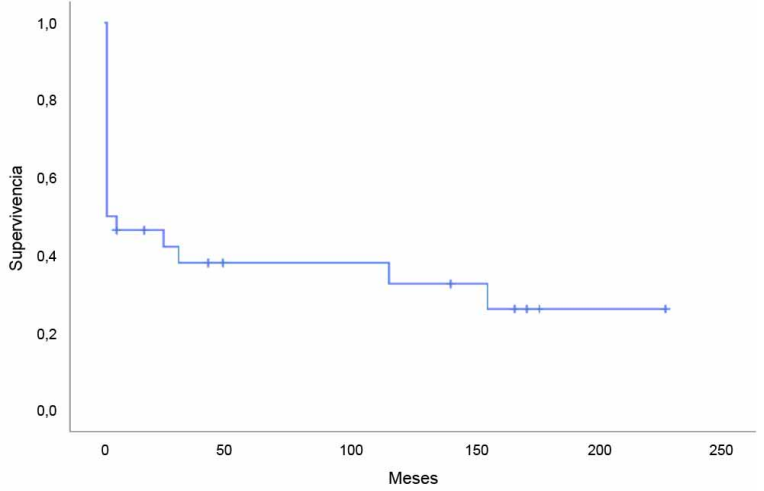

Fig. 4. SVLE para los pacientes de riesgo estándar y alto.

rísticas clínicas, tratamiento y SVde este grupo de niños (as) atendidos en un país en vías de desarrollo y que podría ser utilizada por el resto de las unidades oncológicas de la región en la atención de sus pacientes.

Es un estudio unicéntrico y regional que congrega la totalidad de pacientes con HB, con características clínicas y resultados comparables con otras series.

Comentarios de los resultados obtenidos: El HB se presenta alrededor del año con predominio en hombres (Feng et al.). La edad promedio en esta serie fue de 40 meses, similar a la reportada por otros estudios de la región (Herrera et al., 1999); al igual que la histología epitelial que fue la más frecuente $(42,8 \%)$ (Kremer et al.).

Los valores séricos de AFP son útiles para el diagnóstico, conocer la respuesta al tratamiento y por ende para ayudar a determinar el pronóstico. Su negatividad posterior al tratamiento indica RC (Liu et al.), como en el caso de este estudio; donde el promedio inicial de AFP fue marcadamente elevado $(1055712 \mathrm{ng} / \mathrm{ml})$ y luego del tratamiento clínico y quirúrgico, disminuyó notoriamente (18 ng/ml).

El tratamiento que incluye al menos 7 ciclos de cisplatino y carboplatino con doxorrubicina seguido de cirugía están recomendados (O'Neill et al., 2017). Otras combinaciones, como vincristina e irinotecán también están indicadas en pacientes de riesgo alto (Katzenstein et al., 2017). El objetivo de la QTNA es disminuir o convertir un tumor inicialmente inoperable en operable (Feng et al.). De los diecinueve pacientes que recibieron QTNA, se operaron diez $(52,6 \%)$. Los demás no respondieron favorablemente. Los pacientes operados recibieron de promedio 3 ciclos de quimioterapia postquirúrgico como recomienda la bibliografía (Tannuri et al., 2015; Katzenstein et al., 2019). 
La metástasis pulmonares presente al diagnóstico y el riesgo alto fueron más frecuentes que en otros estu$\operatorname{dios}(27,6 \%$ vs $10,0 \%$ y $69,0 \%$ vs $15,0 \%$ respectivamente) y también son considerados factores de mal pronóstico (la SVAG en estas condiciones son entre 25,0y 50,0\%) (O'Neill et al.; Liu et al.).

La SVAG observada (33,0\%), puede deberse a que el $69,0 \%$ de los pacientes presentaron tumores no resecables y metastásicos al momento del diagnóstico. En países desarrollados se obtienen SVAG de $75,0 \%$ a 82,0 $\%$, teniendo en cuenta que el 73,0 \% de los pacientes en términos generales no tienen enfermedad extra hepática (Feng et al.).

Los pacientes con $\mathrm{HB}$ de riesgo bajo registraron una SV mayor, sin embargo, casi todos los pacientes con diagnóstico de $\mathrm{HB}$ de riesgo alto fallecieron.

Sólo 10 pacientes $(34,5 \%)$ pudieron ser operados, en relación con el $67,0 \%$ de pacientes que son intervenidos regularmente en EE.UU. El hecho de no poder intervenir quirúrgicamente a los pacientes es un factor de mal pronóstico en la SV. Tanto así, que la SVAG en pacientes con resección quirúrgica puede ser de alrededor de 90,0\% mientras que en pacientes no operados, sólo de 35,8\% (Feng et al.). De hecho, la única posibilidad de curación del HB está relacionada con el éxito de la cirugía.

El trasplante hepático está indicado en pacientes con tumores avanzados (Meyers et al.; Liu et al.). Este procedimiento es una alternativa terapéutica que mejora la SV pero no se dispone de esta técnica en el Instituto donde se realizó este estudio, por lo que lamentablemente no se le puede ofrecer a nuestros pacientes.

La mayoría de los pacientes que fueron referidos al Instituto, eran de riesgo alto $(67,9 \%)$ y tenían factores de mal pronóstico reconocidos, como son infiltración bilateral del hígado, infiltración vascular, y metástasis pulmonares (Czauderna et al., 2016; Shi et al., 2017; Liu et al.). En estos pacientes, la SVAG a 5 años es baja (20,0 a 30,0\%) (Feng et al.). Está condición aumentó la morbilidad y la mortalidad y debido a sus bajas posibilidades de curación, motivó que el 25,0 \% de pacientes abandonaran el tratamiento luego de los primeros ciclos de quimioterapia.

Las mejoras en las técnicas quirúrgicas y los protocolos de quimioterapia cada vez mas sofisticados, han hecho que la SV en estos pacientes sean cada vez más altas (Feng et al.); sin embargo, la SV en países en vías de desarollo aún sigue siendo escasa, debido principalmente al diagnóstico tardío. De hecho, estos resultados prácticamente reflejan lo que podría considerarse la evolución natural de la enfermedad ( $\sin$ tratamiento).

A pesar de que la SVLE fue significativamente mejor en pacientes de riesgo estándar que en los de riesgo alto, no podemos sugerir recomendación alguna al respecto, debido al reducido número de pacientes que componen esta serie.

Limitaciones del estudio: se trata de una serie pequeña de pacientes, cuyos resultados se pueden ver comprometidos por los sesgos inherentes a un estudio de carácter retrospectivo; destacando la falta de información de los pacientes que abandonaron el tratamiento. Por otra parte, no se cuenta con algunos recursos para su atención. Sin embargo, los resultados son de interés local porque muestra las limitaciones que tienen los profesionales para enfrentar esta patología con los recursos necesarios.

\section{CONCLUSIÓN}

Los resultados verificados en esta serie de pacientes en términos de SVAG y SVLE, en general y de acuerdo con su extensión; son bajos en relación con otras series internacionales.

GUERRERO, E.; AlVARADO, R.; MONSALVE, P.; PEÑAFIEL, E.; DÍAZ, T.; DUQUE, G.; MANTEROLA, C.; ALBARRACÍN, L. Results of treatment in patients with hepatoblastoma in the Ecuadorian Andes. Int. J. Morphol., 39(6):1737-1742, 2021.

SUMMARY: Hepatoblastoma (HB), is a malignant neoplasm, which originates in the liver. Survival (SV) depends on the extent of disease progression. The objective of this study was to determine differences in overall SV (OS) and disease-free (DFS) in patients with $\mathrm{HB}$, according to the extent of their disease. Case series with follow-up. Patients between 4 and 160 months of age treated at an oncology center in the Ecuadorian Andes (2000-2019) were included. The result variables were affected lobe, lung metastasis, vascular infiltration, PRETEXT stage, risk, histology, alpha-fetoprotein levels (AFP), complete remission (RC), OS and DFS. Descriptive and analytical statistics (Chi2, Fisher's exact and continuity correction) were used. SV analyzes were performed with Kaplan Meier and log-rank curves. In this analysis 28 patients (53.6 $\%$ men), with a median age of 40 months, were studied. Lung metastases and vascular infiltration were verified in $25.0 \%$ and $35.7 \%$ of the cases, respectively. Histology, clinical stage, and high risk were mainly epithelial type (42.8\%), PRETEXT II (50.0 $\%$ ), and high risk (67.8\%), respectively. The mean AFP at diagnosis was $1055712 \mathrm{ng} / \mathrm{ml}$ and 9 patients achieved CR. OS and DFS 
at 19 years were $33.1 \%$ and $26.0 \%$ respectively. According to their extension, the OS and DFS for standard and high risk patients were $50.0 \%$ and $25.4 \%(\mathrm{p}=0.148)$; and $50.0 \%$ and $14.7 \%(\mathrm{p}=$ $0.037)$ respectively. The verified OS and DFS are lower than those reported in other studies. DFS according to its extension, presented a significant difference, however, this result should be considered with caution due to the small number of patients.

KEY WORDS: "Hepatoblastoma"[Mesh]; "Survival"[Mesh]; "Disease-Free Survival"[Mesh]; "Chemotherapy, Adjuvant"[Mesh]; "Surgical Procedures, Operative"[Mesh].

\section{REFERENCIAS BIBLIOGRÁFICAS}

Czauderna, P. \& Garnier, H. Hepatoblastoma: current understanding, recent advances, and controversies. F1000Res., 7:53, 2018.

Czauderna, P.; Haeberle, B.; Hiyama, E.; Rangaswami, A.; Krailo, M.; Maibach, R.; Rinaldi, E.; Feng, Y.; Aronson, D.; Malogolowkin, M.; et al. The Children's Hepatic tumors International Collaboration (CHIC): Novel global rare tumor database yields new prognostic factors in hepatoblastoma and becomes a research model. Eur. J. Cancer, 52:92101,2016

Davenport, K. P.; Blanco, F. C. \& Sandler, A. D. Pediatric malignancies: neuroblastoma, Wilm's tumor, hepatoblastoma, rhabdomyosarcoma, and sacroccygeal teratoma. Surg. Clin. North Am., 92(3):745-67, 2012.

Feng, T. C.; Zai, H. Y.; Jiang, W.; Zhu, Q.; Jiang, B.; Yao, L.; Li, X. Y. \& Wang, Z. M. Survival and analysis of prognostic factors for hepatoblastoma: based on SEER database. Ann. Transl. Med., 7(20):555, 2019.

Guo, F.; Ru, Q.; Zhang, J.; He, S.; Yu, J.; Zheng, S. \& Wang, J. Inflammation factors in hepatoblastoma and their clinical significance as diagnostic and prognostic biomarkers. J. Pediatr. Surg., 52(9):1496-502, 2017.

Herrera, J. M.; Barriga, F.; Harris, P.; Ronco, R.; García, C. \& Rossi, R. Multidisciplinary management of malignant hepatic tumors in children: a recent national experience. Rev. Med.Chil., 127(11):1351-8, 1999.

Katzenstein, H. M.; Furman, W. L.; Malogolowkin, M. H.; Krailo, M. D.; McCarville, M. B.; Towbin, A. J.; Tiao, G. M.; Finegold, M. J.; Ranganathan, S.; Dunn, S. P.; et al. Upfront window vincristine/ irinotecan treatment of high-risk hepatoblastoma: A report from the Children's Oncology Group AHEP0731 study committee. Cancer, 123(12):2360-7, 2017.

Katzenstein, H. M.; Langham, M. R.; Malogolowkin, M. H.; Krailo, M. D.; Towbin, A. J.; McCarville, M. B.; Finegold, M. J.; Ranganathan, S.; Dunn, S.; McGahren, E. D.; et al. Minimal adjuvant chemotherapy for children with hepatoblastoma resected at diagnosis (AHEP0731): a Children's Oncology Group, multicentre, phase 3 trial. Lancet Oncol., 20(5):719-27, 2019

Kiruthiga, K. G.; Ramakrishna, B.; Saha, S. \& Sen, S. Histological and immunohistochemical study of hepatoblastoma: correlation with tumour behaviour and survival. J. Gastrointest. Oncol., 9(2):326-37, 2018.

Kremer, N.; Walther, A. E. \& Tiao, G. M. Management of hepatoblastoma: an update. Curr. Opin. Pediatr., 26(3):362-9, 2014.

Liu, A. P. Y.; Ip, J. J. K.; Leung, A. W. K.; Luk, C. W.; Li, C. H.; Ho, K. K. H.; Lo, R.; Chan, E. K. W.; Chan, A. C. Y.; Chung, P. H. Y.; et al. Treatment outcome and pattern of failure in hepatoblastoma treated with a consensus protocol in Hong Kong. Pediatr. Blood Cancer, 66(1):e27482, 2019

Manterola, C. \& Astudillo, P. Checklist for reporting of descriptive observational studies. MINCIR initiative. Int. J. Morphol., 31(1):11520, 2013.
Martinez, F.; Abril, L. \& Pérez, L. Epidemiología del Cancer en el Cantón Cuenca 2005-2009, Sexto Informe. Cuenca, SOLCA Núcleo de Cuenca, 2015.

Meyers, R. L.; Tiao, G.; de Ville de Goyet, J.; Superina, R. \& Aronson, D. C. Hepatoblastoma state of the art: pre-treatment extent of disease, surgical resection guidelines and the role of liver transplantation. Curr. Opin. Pediatr, 26(1):29-36, 2014.

O'Neill, A. F.; Towbin, A. J.; Krailo, M. D.; Xia, C.; Gao, Y.; McCarville, M. B.; Meyers, R. L.; McGahren, E. D.; Tiao, G. M.; Dunn, S. P.; et al. Characterization of pulmonary metastases in children with hepatoblastoma treated on Children's Oncology Group Protocol AHEP0731 (The Treatment of Children With All Stages of Hepatoblastoma): a report from the Children's Oncology Group. J. Clin. Oncol., 35(30):3465-73, 2017.

Sharma, D.; Subbarao, G. \& Saxena, R. Hepatoblastoma. Semin. Diagn. Pathol., 34(2):192-200, 2017

Shi, Y.; Commander, S. J.; Masand, P. M.; Heczey, A.; Goss, J. A. \& Vasudevan, S. A. Vascular invasion is a prognostic indicator in hepatoblastoma. J. Pediatr. Surg., 52(6):956-61, 2017.

Tannuri, A. C. A.; Cristofani, L. M.; Teixeira, R. A. P.; Odone Filho, V. \& Tannuri, U. New concepts and outcomes for children with hepatoblastoma based on the experience of a tertiary center over the last 21 years. Clinics (Sao Paulo), 70(6):387-92, 2015.

van Dalen, E. C.; Raphaël, M. F.; Caron, H. N. \& Kremer, L. C. M. Treatment including anthracyclines versus treatment not including anthracyclines for childhood cancer. Cochrane Database Syst. Rev., (9):CD006647, 2014.

Weldon, C. B.; Madenci, A. L.; Tiao, G. M.; Dunn, S. P.; Langham, M. R.; McGahren, E. D.; Ranganathan, S.; López-Terrada, D. H.; Finegold, M. J.; Malogolowkin, M. H.; et al. Evaluation of the diagnostic biopsy approach for children with hepatoblastoma: A report from the children's oncology group AHEP 0731 liver tumor committee. J. Pediatr. Surg., 55(4):655-9, 2020.

\author{
Dirección para Correspondencia: \\ Dr. Carlos Manterola \\ CEMyQ \\ Universidad de La Frontera \\ Temuco \\ CHILE
}

E-mail: carlos.manterola@ufrontera.cl

Recibido : 27-08-2021

Aceptado :26-09-2021 